\title{
ON THE ZEROS OF DIRICHLET L-FUNCTIONS. I
}

BY

\section{AKIO FUJII( $\left.{ }^{1}\right)$}

\section{ABSTRACT. A mean value theorem for arg $L(1 / 2+i(t+h), x)-$} $\arg L(1 / 2+i t, x)$ is established. This yields mean estimates for the number of zeros of $L(s, x)$ in small boxes.

1. Statement of results. As is customary we let $S(t, \chi)=\pi^{-1}$ arg $L(1 / 2+i t, \chi)$, where the argument is determined by continuous variation on the half-line $\sigma+i t$, $\sigma \geq 1 / 2$. If $t$ is the ordinate of a zero then we put

$$
s(t, \chi)=1 / 2(s(t+0, \chi)+S(t-0, \chi))
$$

We assume throughout that $\chi$ is a primitive character mod $q$. The zeta function arises from the principal character with $q=1$; thus $\zeta(s)=L\left(s, \chi_{0}\right)$, and we write $S(t)=S\left(t, \chi_{0}\right)$.

In $1944 \mathrm{~A}$. Selberg [2] demonstrated that

$$
\int_{T}^{T+H} S(t)^{2 k} d t=c_{k} H(\log \log T)^{k}+O_{k}\left(H(\log \log T)^{k-1 / 2}\right)
$$

provided that $T^{1 / 2+a} \leq H \leq T, 0<a \leq 1 / 2$, where

$$
c_{k}=\frac{(2 k) !}{(2 \pi)^{2 k} k !}
$$

We modify $\Lambda$. Selberg's approach to obtain our

Main Theorem. Let $a_{1}, a_{2}$ be fixed, $0<a_{i} \leq 1 / 2$ for $i=1,2$. Let $\chi$ be a primitive character $(\bmod q)$, and suppose that $q \leq T^{1 / 4-a_{1}}, T^{1 / 2+a_{2}} \leq H \leq T$, and that $0<b \leq\left(H-(H / \sqrt{T})^{1 / 8}\right)$. Then

$$
\begin{array}{r}
\int_{T}^{T+H}(S(t+h, \chi)-S(t, \chi))^{2 k} d t=c_{k} H(2 \log (2+h \log T))^{k} \\
\cdot\left(1+O\left((A k)^{3 k}(\log (2+h \log T))^{-1 / 2}\right)\right),
\end{array}
$$

where $A>0$ is a suitable absolute constant, and $c_{k}$ is given by (1).

Received by the editors October 1, 1973.

AMS (MOS) subject classifications (1970). Primary $10 \mathrm{H} 05,10 \mathrm{H} 10$. of zeros.

$K e y$ words and phrases. Riemann zeta function, Dirichlet $L$-functions, distribution

(1) Supported in part by National Science Foundation grant GP-36418X1. 
Let $N(T, \chi)$ denote the number of zeros $\rho=\beta+i y$ of $L(s, \chi)$ with $0<\beta<1$, $0 \leq \gamma \leq T$, zeros on the boundary being counted with weight one-half. It is well known (see [3]) that if $T \geq 0$ then

$$
N(T, \chi)=\frac{T}{2 \pi} \log \frac{q T}{2 \pi}-\frac{T}{2 \pi}-\frac{\chi(-1)}{8}+S(T, \chi)-S(0, \chi)+O\left(\frac{1}{T+1}\right)
$$

Thus we easily derive the following

Corollary. Under the above hypothesis,

$$
\begin{aligned}
& \int_{T}^{T+H}(N(t+h, \chi)-N(t, \chi))^{2 k} d t \\
&= H\left(\frac{h}{2 \pi} \log q T\right)^{2 k}\left(1+O\left((A k)^{4 k} \frac{(\log (2+h \log T))^{1 / 2}}{2+h \log T}\right)\right) \\
& \text { unless } h \log T \rightarrow 0 \text { as } T \rightarrow \infty, \\
&=O\left((A k)^{4 k} H\right) \text { if } h \log T \rightarrow 0 \text { as } T \rightarrow \infty
\end{aligned}
$$

where $A>0$ is a suitable absolute constant.

We may remark here that implicit constants involved in $O$ or $\ll$ in this paper do not depend on $q, k$ and $b$ unless it is written explicitly like $O_{k}$.

The author would like to express his deep gratitude to Professor Patrick X. Gallagher for his constant encouragement and many valuable suggestions. Thanks are also due to Professor Hugh L. Montgomery who kindly read the manuscript and suggested various simplications for publication.

2. Lemmas. The condition $q \leq T^{1 / 4-a_{1}}$ in the Main Theorem comes from

Lemma 1. Let $a_{1}, a_{2}$ be fixed, $0<a_{i} \leq 1 / 2$ for $i=1,2$. Let $\chi$ be a primitive character $(\bmod q)$, and suppose that $q \leq T^{1 / 4-a_{1}}, T^{1 / 2+a_{2}} \leq H \leq T$. Then

$$
N_{\chi}(\sigma, T+H)-N_{\chi}(\sigma, T)=O\left(H(H / \sqrt{T})^{(1-2 \sigma) / 4} \log T\right)
$$

uniformly for $\sigma$ in $1 / 2 \leq \sigma \leq 1$, where $N_{\chi}(\sigma, T)$ denotes the number of zeros $\beta+i \gamma$ of $L(s, \chi)$ with $\sigma<\beta$ and $0<\gamma<T$.

Proof. We will follow the argument of A. Selberg (cf. [2]) and we will state only the results in each step.

(i) We use A. F. Lavrik's approximate functional equation in the following form (cf. [1])

$$
L(s, \chi)=\sum_{n \leq x} \frac{\chi(n)}{n^{s}}+\epsilon(\chi)\left(\frac{q}{\pi}\right)^{1 / 2-s} \frac{\Gamma(1 / 2(1-s+a))}{\Gamma(1 / 2(s+a))} \sum_{n \leq y} \frac{\overline{\chi(n)}}{n^{1-s}}+R_{x y},
$$

where 


$$
s=\sigma+i t, 0<\sigma<1, a=1 / 2(1-\chi(-1)), x=\Delta \sqrt{q|t| / 2 \pi}, y=\frac{1}{\Delta} \sqrt{q|t| / 2 \pi},
$$

$f(\chi)=(-1)^{a} \frac{1}{\sqrt{q}} \sum_{n=1}^{q} \chi(n) e^{2 \pi i(n / q)}$ and $R_{x y} \ll \sqrt{q}\left(y^{-\sigma}+x^{\sigma-1}\left(q t^{\prime}\right)^{1 / 2-\sigma}\right) \log ^{2}|t|$

with $t^{\prime}=\max (|t|, 1)$.

(ii) Using (i) if $T>10, \sqrt{T} \leq H \leq T, \mu_{1}$ and $\mu_{2}$ are positive coprime integers less than $T / 10$, we have for $1 / 2<\sigma \leq 1$,

$$
\begin{aligned}
\int_{T}^{T+H} \mid L(\sigma+i t & \chi)\left.\right|^{2}\left(\frac{\mu_{1}}{\mu_{2}}\right)^{i t} d t \\
= & \chi\left(\mu_{1}\right) \bar{\chi}\left(\mu_{2}\right) \int_{T}^{T+H}\left\{\left(\mu_{1} \mu_{2}\right)-\sigma \zeta(2 \sigma)+\left(\mu_{1} \mu_{2}\right)^{\sigma-1} \tau^{2-4 \sigma} \zeta(2-2 \sigma)\right\} d t \\
& +O\left((q T)^{1-\sigma} \mu_{1} \mu_{2} \log ^{2}(q T)\right) \\
& +O\left(q^{1-\sigma} H T^{-2}\left(\left(\frac{\mu_{1}}{\mu_{2}}\right)^{1 / 2}+\left(\frac{\mu_{2}}{\mu_{1}}\right)^{1 / 2}\right) \log (q T) \cdot \log T\right)
\end{aligned}
$$

where $r=\sqrt{q t / 2 \pi}$.

(iii) For $T^{1 / 2+a} \leq 4 \leq T, 0<a_{2} \leq 1 / 2, \xi=(H / \sqrt{T})^{1 / 4}, 1 / 2+1 / \log \xi \leq \sigma \leq 1$ and $q \leq T^{1 / 4-a_{1}}$,

$$
\int_{T}^{T+H}|L(\sigma+i t, \chi) \psi(\sigma+i t, \chi)|^{2} d t<H+O\left(H(H / \sqrt{T})^{(1-2 \sigma) / 4}\right),
$$

where $\psi(s, \chi)=\Sigma_{\nu<\xi}\left(\lambda_{\nu} / \nu^{s}\right) \bar{\chi}(\nu)$ and $\lambda_{\nu}$ is defined as follows: for $\xi>1$ and $1 / 2<\sigma \leq 1$ and $1 \leq \nu<\xi$

$$
\lambda_{\nu}=\frac{\nu^{2 \sigma}}{\sum_{\rho<\xi} \mu^{2}(\rho) / \phi_{2 \sigma}(\rho)} \sum_{\rho<\xi / \nu} \frac{\mu(\rho \nu) \mu(\rho)}{\phi_{2 \sigma}(\rho \nu)}
$$

with $\phi_{\gamma}(\rho)=\rho^{\gamma} \Sigma_{d \mid \rho} \mu(d) / d^{\gamma}$ for a positive integer $\rho$ and $\mu(d)$ is the Möbius function.

Proof. Same computation as A. Selberg (cf. [2]) leads to the following.

$$
\begin{aligned}
& \int_{T}^{T+H}|L(\sigma+i t, \chi) \psi(\sigma+i t, \chi)|^{2} d t \\
& <H+O\left(H \xi^{1-2 \sigma}\right)+O\left((q T)^{1-\sigma} \xi^{4-2 \sigma} \log ^{2}(q T) \cdot \log ^{2} T\right) \\
& \quad+O\left(q^{1-\sigma} H T^{-\sigma / 2} \log (q T) \cdot \log ^{4} T\right) \\
& <H+O\left(H(H / \sqrt{T})^{(1-2 \sigma) / 4}\right)+O\left(q^{1-\sigma} H T^{1 / 2-\sigma-\left(2 a_{1}-1\right) / 8} \cdot \log ^{2} T \cdot \log ^{2}(q T)\right) \\
& \quad+O\left(q^{1-\sigma} H T^{1 / 8-\sigma / 2} \log ^{4} T \cdot \log (q T)\right) .
\end{aligned}
$$


Hence if $q \leq T^{\left(1 / 4-a_{1}\right)}$ with $a_{1}>0$, the last two remainder terms become $O\left(H(H / \sqrt{T})^{(1-2 \sigma) / 4}\right)$ and we get the conclusion.

(iv) Using Littlewood's lemma (cf. p. 106 in [2]) we get our conclusion. Q.E.D.

Let $a(p)$ be a real or complex valued function of prime numbers $p$. For simplicity we write

$$
F_{a}(x)=\sum_{p \leq x} \frac{|d(p)|^{2 a}}{p^{a}}
$$

for a real number $a$.

Lemma 2. Assume that $F_{a}(x) \ll 1$ for $a \geq 2$. Then

$$
\begin{aligned}
\sum_{p_{i}<x}^{*} \frac{a\left(p_{1}\right) d\left(p_{2}\right) \cdots a\left(p_{k}\right) \overline{d\left(p_{k+1}\right)} \cdots \overline{a\left(p_{2 k}\right)}}{\sqrt{p_{1} p_{2} \cdots p_{k} p_{k+1} \cdots p_{2 k}}} \\
\quad= \begin{cases}k ! F_{1}(x)^{k}+O\left(k ! F_{1}(x)^{o v(k-2)}\right) \text { if } F_{1}(x) \rightarrow \infty \text { as } x \rightarrow \infty, \\
O(k !) \text { if } F_{1}(x) \ll 1,\end{cases}
\end{aligned}
$$

where in $\Sigma_{p_{i}<x}^{*}$ we sum over all primes $p_{1}, \cdots, p_{2 k}$ satisfying $p_{1} \cdots p_{k}=$ $p_{k+1} \cdots p_{2 k}$ and $p_{i}<x$ for $i=1,2, \cdots, 2 k$, and $\operatorname{ov}(k-2)=\max \{0, k-2\}$.

Proof. The expressions of $k$ by the sum of $n$ natural numbers can be ordered lexicographically when we identify two expressions up to permutations. Let $\sigma_{k}(n)$ be the number of such expressions. Let $\Phi\left(k-b, \nu_{b}\right)$ be the $\nu_{b}$ th expression $\left(c_{1}, c_{2}, \cdots, c_{k-b}\right)$ of $k$ by the sum of $k-b$ natural numbers; $k=c_{1}+c_{2}+\cdots+$ $c_{k-b}$. Let

$$
A_{k-b, \nu_{b}}=\sum_{p_{i}<x} \frac{\left|a\left(p_{1}\right)\right|^{2 c_{1}}\left|a\left(p_{2}\right)\right|^{2 c_{2}} \cdots\left|a\left(p_{k-b}\right)\right|^{2 c_{k-b}}}{p_{1}^{c_{1}} \cdots p_{k-b}^{c_{k-b}}}
$$

for $\nu_{b}=1,2, \cdots, \sigma_{k}(k-b)$, where in $\Sigma_{p_{i}<x}^{\prime}$ we sum over all $p_{1}, \cdots, p_{k-b}$ with $p_{i}<x$ for $i=1, \cdots, k-b$ whenever $p_{1}, \cdots, p_{k-b}$ are all different, and $\left(c_{1}, c_{2}, \cdots, c_{k-b}\right)=\Phi\left(k-b, v_{b}\right)$. Also let

$$
\begin{aligned}
\bar{A}_{k-b, \nu_{b}} & =F_{c_{1}}(x) F_{c_{2}}(x) \cdots F_{c_{k-b}}(x) \\
& =\left(\sum_{p<x} \frac{|a(p)|^{2 c_{1}}}{p^{c_{1}}}\right) \cdots\left(\sum_{p<x} \frac{|a(p)|^{2 c_{k-b}}}{p^{c_{k-b}}}\right) .
\end{aligned}
$$


Now

$$
\sum_{p_{i}<x}^{*} \frac{a\left(p_{1}\right) a\left(p_{2}\right) \cdots a\left(p_{k}\right) \overline{a\left(p_{k+1}\right)} \cdots \overline{a\left(p_{2 k}\right)}}{\sqrt{p_{1} p_{2} \cdots p_{2 k}}}=\sum_{p_{i}<x}^{*} \frac{\left|a\left(p_{1}\right)\right|^{2} \cdots\left|a\left(p_{k}\right)\right|^{2}}{p_{1} \cdots p_{k}}
$$

(1)

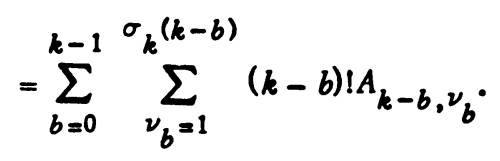

We remark that $\bar{A}_{k-b, \nu_{b}}=A_{k-b, \nu_{b}}+\sum_{b^{\prime}=b+1}^{k-1} \sum_{\nu_{b^{\prime}}=1}^{\sigma} A_{k-b^{\prime}, \nu_{b^{\prime}}}^{\left(k-b^{\prime}\right)}$ Substituting this into the above, we get

$$
(1)=k ! F_{1}(x)^{k}+\sum_{b=1}^{k-1}(b-k) \cdot(k-b) ! \sum_{\nu_{b}=1}^{\sigma_{k}(k-b)} \bar{A}_{k-b, \nu_{b}}
$$

since $\bar{A}_{k, \nu_{0}}=\left(\Sigma_{p<x}|a(p)|^{2} / p\right)^{k}=F_{1}(x)^{k}$. Now by the assumption if $F_{1}(x) \rightarrow \infty$ as $x \rightarrow \infty$

(2)

$$
\bar{A}_{k-b, \nu_{b}} \ll F_{1}(x)^{o v(k-2)} \text { for } 1 \leq b \leq k-1 .
$$

Hence it is enough to show that

$$
(b-k) \cdot(k-b) ! \ll \sigma_{k}(k-b) \ll(k-1) !
$$

for $b=1,2, \cdots, k-1$. Moreover it is enough to estimate this for sufficiently big $k$. We define $\sigma_{k}^{\prime}(n)$ by

$$
\frac{x^{n}}{(1-x)^{n}}=\sum_{k=n}^{\infty} \sigma_{k}^{\prime}(n) x^{n}
$$

then $\sigma_{k}(n) \leq \sigma_{k}^{\prime}(n)$. Since

$$
\frac{x^{n}}{(1-x)^{n}}=\sum_{k \geq n} \frac{(k-1) !}{(n-1) !(k-n) !} x^{k},
$$

$\sigma_{k}(n) \leq \sigma_{k}^{\prime}(n)=(k-1) ! /(n-1) !(k-n) !$. Now $(3)$ is clear for $b \ll 1$ or $k-b<1$. In other cases since $(k-b)^{2} \leq b$ ! for sufficiently big $k$,

$$
\begin{aligned}
\left|(b-k) \cdot(k-b) ! \sigma_{k}(k-b)\right| & \leq \frac{(k-1) !}{(k-b-1) !(k-k+b) !}(k-b) ! \cdot(k-b) \\
& \leq(k-1) !(k-b)^{2} / b ! \leq(k-1) ! .
\end{aligned}
$$

Hence by (2) and (3) $(1)=k ! F_{1}(x)^{k}+O\left(k ! F_{1}(x)^{o v(k-2)}\right)$. If $F_{1}(x) \ll 1$, $\bar{A}_{k-b, \nu_{b}} \ll 1$ for $0 \leq b \leq k-1$. Hence we get our conclusion. Q.E.D. 
Lemma 3. Assume that $F_{a}(x) \ll 1$ for $\alpha \geq 2$ and $F_{1 / 2}(x) \ll<x^{c}$ with some positive c. Then for $x=T^{\left(a_{2}+1 / 2\right) / 2 k(c+1)}$

$$
\begin{aligned}
\int_{T}^{T+H} \mid & \left.\operatorname{Im} \sum_{p<x} \frac{a(p)}{p^{1 / 2+i t}}\right|^{2 k} d t \\
& = \begin{cases}\frac{2 k !}{2^{2 k} k !} H F_{1}(x)^{k}+O\left(\frac{2 k !}{2^{2 k} k !} H F_{1}(x)^{\circ v(k-2)}\right) & \text { if } F_{1}(x) \rightarrow \infty \text { as } x \rightarrow \infty, \\
O\left(k^{k} H\right) & \text { if } F_{1}(x) \ll 1, \\
O\left(k^{k} A^{2 k} H\right) & \text { always, }\end{cases}
\end{aligned}
$$

where $A$ satisfies $|a(p)| \leq A \log p / \log x$ for any $p \leq x$.

Proof (First case). We write

$$
\tau=\sum_{p<x} \frac{d(p)}{p^{1 / 2+i t}}
$$

and

$$
\operatorname{Im} \sum_{p<x} \frac{d(p)}{p^{1 / 2+i t}}=\frac{r-\bar{\tau}}{2 i}
$$

and

$$
\begin{aligned}
& \left|\operatorname{Im} \sum_{p<x} \frac{a(p)}{p^{1 / 2+i t}}\right|^{2 k}=\frac{1}{2^{2 k}} \sum_{b=0}^{2 k}(-1)^{k-b}\left(\begin{array}{c}
2 k \\
b
\end{array}\right) \cdot \int_{T}^{T+H} b_{r} \bar{r}^{2 k-b} d t \\
& \quad=\frac{1}{2^{2 k}} \sum_{b=0}^{2 k}(-1)^{k-b}\left(\begin{array}{c}
2 k \\
b
\end{array}\right) \sum_{p<x} \frac{a\left(p_{1}\right) \cdots a\left(p_{b}\right) \overline{a\left(p_{b+1}\right)} \cdots \overline{a\left(p_{2 k}\right)}}{\sqrt{p_{1} p_{2} \cdots p_{2 k}}} \int_{T}^{T+H}\left(\frac{p_{b+1} \cdots p_{2 k}}{p_{1} \cdots p_{b}}\right)^{i t} d t .
\end{aligned}
$$

If $p_{b+1} \cdots p_{2 k} \neq p_{1} \cdots p_{b}$,

$$
\int_{T}^{T+H}\left(\frac{p_{b+1} \cdots p_{2 k}}{p_{1} \cdots p_{b}}\right)^{i t} d t=O\left(1 / \log \frac{p_{b+1} \cdots p_{2 k}}{p_{1} \cdots p_{b}} \mid\right)=O\left(x^{2 k}\right)
$$

Hence for $b \neq k$,

$$
\int_{T}^{T+H}{ }_{\tau}^{b} \bar{\tau}^{-2 k-b} d t=O\left(x^{2 k} F_{1 / 2}(x)^{2 k}\right)=O\left(x^{2 k(1+c)}\right)=O(H) .
$$

If $b=k$, we get by Lemma 1

$$
\begin{aligned}
\int_{T}^{T+H}||^{2 k} d t & =H \sum_{p_{i}<x}^{*} \frac{a\left(p_{1}\right) \cdots a\left(p_{k}\right) \overline{a\left(p_{k+1}\right)} \cdots \overline{a\left(p_{2 k}\right)}}{p_{1} \cdots p_{k}}+O(H) \\
& =k ! H F_{1}(x)^{k}+O\left(H k ! F(x)^{o v(k-2)}\right)
\end{aligned}
$$


where the meaning of $\Sigma_{p_{i}<x}^{*}$ is the same as in Lemma 1. Hence

$$
\begin{aligned}
\int_{T}^{T+H}\left|\operatorname{Im} \sum_{p<x} \frac{a(p)}{p^{1 / 2+i t}}\right|^{2 k} d t= & \frac{1}{2^{2 k}}\left(\begin{array}{c}
2 k \\
k
\end{array}\right) H k ! F_{1}(x)^{k} \\
& +O\left(\frac{1}{2^{2 k}}\left(\begin{array}{c}
2 k \\
k
\end{array}\right) H k ! F_{1}(x)^{o v(k-2)}\right) \\
& +O\left(\frac{1}{2^{2 k}} \sum_{b=0 ; b \neq k}^{2 k}\left(\begin{array}{c}
2 k \\
b
\end{array}\right) H\right) \\
= & \frac{2 k !}{2^{2 k} k !} H F_{1}(x)+O\left(\frac{2 k !}{2^{2 k} k !} H F_{1}(x)^{o v(k-2)}\right) .
\end{aligned}
$$

(Other cases). For $b \neq k, \int_{T}^{T+H_{\tau} b} \bar{\tau}^{2 k-b} d t=O(H)$ as above. For $b=k$,

$$
\int_{T}^{T+H}|\tau|^{2 k} d t=H \sum_{p_{i}<x}^{*} \frac{a\left(p_{1}\right) \cdots a\left(p_{k}\right) \overline{a\left(p_{k+1}\right)} \cdots \overline{a\left(p_{2 k}\right)}}{p_{1} \cdots p_{k}}+O(H)=O(k ! H)
$$

by Lemma 1 . Hence we get the second part. The third part comes from Lemma 4 below. Q.E.D.

For $x \geq 2$ we define (see p. 47 of [3]) a number $\sigma_{x}(t)$ by

$$
\sigma_{\chi}(t) \equiv \sigma_{\chi}(x, t)=1 / 2+2 \underset{\rho}{\operatorname{Max}}(\beta-1 / 2,2 / \log x),
$$

where $\rho=\beta+i \gamma$ runs through the zeros of $L(s, \chi)$ for which $|t-\gamma| \leq$ $x^{3(\beta-1 / 2)} / \log x$. Now modifying A. Selberg's arguments (i.e., Lemma 12 on p. 121 of [2] and Lemma 13 on p. 123 of [2]) we get the following two lemmas. We omit these proofs. We use Lemma 1 to prove Lemma 4. The condition on $b$ in the Main Theorem comes from Lemma 4.

Lemma 4. Under the same hypothesis as Lemma 1 , for $\xi$ satisfying $1 \leq \xi \leq$ $x^{8 k}$ and $x^{3} \xi^{2} \leq(H / \sqrt{T})^{1 / 4}$, for $\nu$ in $0 \leq \nu \leq 8 k$, and for $b=h(T) \leq H-(H / \sqrt{T})^{1 / 8}$,

$$
\int_{T}^{T+H}\left(\sigma_{\chi}(t+h)-1 / 2\right)^{\nu} \xi^{\sigma_{\chi}(t+b)-1 / 2} d t \ll \frac{H A_{1}^{k} \nu !}{(\log x)^{\nu}},
$$

where $A_{1}$ is some positive absolute constant.

Lemma 5. Let $H>1,1<y \leq H^{1 / k}$ and $|a(p)|<A \log p / \log y$ for $p<y$ and $\left|a^{\prime}(p)\right| \leq B$ for $p<y$. Then

$$
\int_{0}^{H}\left|\sum_{p<y} \frac{a(p)}{p^{1 / 2+i t}}\right|^{2 k} d t=O\left(\left(A^{2} k\right)^{k} A_{1}^{k} H\right)
$$


and

$$
\int_{0}^{H}\left|\sum_{p<y} \frac{a^{\prime}(p)}{p^{1+2 i t}}\right|^{2 k} d t=O\left(\left(B^{2} k\right)^{k} A_{1}^{k} H\right)
$$

where $A_{1}$ 's are some positive absolute constants.

3. Proof of theorem. We need the following expression for $x \geq 2$ due to $A$. Selberg (cf. [3]):

$$
S(t, \chi)-\frac{1}{\pi} \operatorname{Im} \sum_{p<x^{3}} \frac{\chi(p)}{p^{1 / 2+i t}}=\sum_{i=1}^{s} O\left(B_{i}(t)\right)
$$

where

$$
\begin{gathered}
B_{1}(t)=\left|\sum_{p<x} \frac{\Lambda(p)-\Lambda_{x}(p)}{p^{1 / 2+i t} \log p} \cdot \chi(p)\right|, \quad B_{2}(t)=\left(\sigma_{x}(t)-1 / 2\right) \log x, \\
B_{3}(t)=\left(\sigma_{x}(t)-1 / 2\right) \log (q(1+|t|)), \quad B_{4}(t)=\left|\sum_{p<x} \frac{\Lambda_{x}\left(p^{2}\right) \chi\left(p^{2}\right)}{2 p^{1+2 i t} \log p}\right|, \\
B_{5}(t)=\left(\sigma_{x}(t)-1 / 2\right) x{ }^{\sigma_{x}(t)-1 / 2} \int_{1 / 2}^{\infty} x^{1 / 2-\sigma}\left|\sum_{p<x^{3}} \frac{\Lambda_{x}(p) \log (x p)}{p^{\sigma+i t}} \chi(p)\right| d \sigma
\end{gathered}
$$

and

$$
\begin{aligned}
\Lambda_{x}(n) & =\Lambda(n) & & \text { if } 1 \leq n \leq x, \\
& =\Lambda(n) \frac{\left(\log x^{3} / n\right)^{2}-2\left(\log x^{2} / n\right)^{2}}{2(\log x)^{2}} & & \text { if } x \leq n \leq x^{2}, \\
& =\Lambda(n) \frac{\left(\log x^{3} / n\right)^{2}}{2(\log x)^{2}} & & \text { if } x^{2} \leq n \leq x^{3} .
\end{aligned}
$$

For simplicity we write

$$
\|f\|=\left(\int_{T}^{T+H}|f(t)|^{2 k} d t\right)^{1 / 2 k}
$$

We use the above expression for $x=T^{a_{2} / 60 k}$.

Now

$$
A_{x^{3}}(t) \stackrel{\text { def }}{=} S(t+h, \chi)-S(t, \chi)-f_{x^{3}}(t)=\sum_{i=1}^{5} O\left(B_{i}(t+h)\right)+\sum_{i=1}^{5} O\left(B_{i}(t)\right),
$$


where $f_{x^{3}}(t)=\pi^{-1} \operatorname{Im} \Sigma_{p<x^{3}} a(p) / p^{(1 / 2+i t)}$ with $a(p)=\chi(p)\left(e^{-i h \log p}-1\right)$. By Lemma $5\left\|B_{i}(t+b)\right\| \ll k^{1 / 2} H^{1 / 2 k}$ and $\left\|B_{i}(t)\right\| \ll k^{1 / 2} H^{1 / 2 k}$ for $i=1$ and 4. By Lemma 4 with $\xi=1$ and by the assumption on $q,\left\|B_{i}(t+b)\right\| \ll k^{1 / 2} H^{1 / 2 k}$ and $\left\|B_{i}(t)\right\| \ll k^{2} H^{1 / 2 k}$ for $i=2$ and 3. By Lemmas 4 and 5 as on p. 126 of [2],

$$
\left\|B_{s}(t+h)\right\| \ll k^{3 / 2} H^{1 / 2 k} \text { and }\left\|B_{s}(t)\right\| \ll k^{3 / 2} H^{1 / 2 k} \text {. }
$$

Hence for $x=T^{a_{2} / 60 k},\left\|A_{x^{3}}(t)\right\| \ll k^{2} H^{1 / 2 k}$. For $T^{a / 20 k} \leq x \leq H^{1 / k}$,

$$
\begin{aligned}
& \left\|A_{x}(t)\right\| \leq\left\|A_{T^{a} a^{2} / 20 k}(t)\right\|+\left\|\sum_{T^{a_{2} / 20 k} \leq p \leq x} \frac{a(p)}{p^{1 / 2+i t}}\right\| \\
& \ll k^{2} H^{1 / 2 k}+k^{1 / 2} H^{1 / 2 k} \ll k^{2} H^{1 / 2 k} \text { by Lemma } 5 \text {. }
\end{aligned}
$$

Hereafter we take $x=T^{\left(a_{2}+1 / 2\right) / 2 k(c+1)}$, where $c$ is some nonnegative constant satisfying $F_{1 / 2}(x)=\Sigma_{p<x} a(p) / \sqrt{p}<<x^{c}$.

Now in using Lemma 3 in our case

$$
\begin{aligned}
F_{1}(x) & =\sum_{p<x} \frac{\left|e^{-i h \log p}-1\right|^{2}}{p}=2 \sum_{p<x} \frac{1-\cos (h \log p)}{p} \\
& =2 \sum_{p<x} \frac{1-\cos (h \log p) \log p}{\log p}=2 \int_{1}^{x} \frac{1-\cos (h \log \xi)}{\log \xi} d(\log \xi+O(1))
\end{aligned}
$$

since $\Sigma_{p<\xi} \log p / p=\log \xi+O(1)$. Hence

$$
F_{1}(x)=2 \int_{0}^{b \log x} \frac{1-\cos u}{u} d u+O(1)=2 \log (b \log x)+O(1) .
$$

Hence if $b \log T \rightarrow \infty$ as $T \rightarrow \infty$, then $F_{1}(x) \rightarrow \infty$ and by Lemma 3

Hence

$$
\left\|f_{x}(t)\right\|^{2 k}=c(k) H F_{1}(x)^{k}+O\left(c(k) H F_{1}(x)^{o v(k-2)}\right) \text {. }
$$

$$
\begin{aligned}
\|S(t+h, \chi)-S(t, \chi)\|^{2 k}=\left\|A_{x}(t)+f_{x}(t)\right\|^{2 k} \\
\quad=c(k) H(2 \log (h \log T))^{k}+O\left((A k)^{4 k} H(\log (h \log T))^{k-1 / 2}\right)
\end{aligned}
$$

if $b \log T \rightarrow \infty$ as $T \rightarrow \infty$.

If $b \log T \ll 1$, using Lemma 3 again we get similarly

$$
\|S(t+h, \chi)-S(t, \chi)\|^{2 k} \ll(A k)^{4 k} H .
$$

Hence we get our conclusion. Q.E.D. 
4. Concluding remarks. We can get mean estimates of $\sum_{m=0}^{L} \Sigma_{x} b(m) g(\chi) S(t+b m, \chi)$ similarly as we did in $\$ 3$, where $b(0), \cdots, b(L)$ are real numbers and $g(\chi)$ is a complex number for a character $\chi \bmod q$. Instead of describing a general theorem to this, we will give only some examples of this. We always assume that $q \leq T^{1 / 4-a_{1}}$ and $T^{1 / 2+a_{2}} \leq H \leq T$ for a fixed $a_{1}$ and $a_{2}$ in $0<a_{i} \leq 1 / 2$ for $i=1,2$.

(i)

$$
\int_{T}^{T+H}\left(\sum_{\chi}^{\prime} S(t, \chi)\right)^{2 k} d t=c(k) H(\phi(q)-1)^{2 k}(\log \log T)^{k}
$$

$$
+O_{k}\left(H(\phi(q)-1)^{2 k-1}(\log \log T)^{k-1 / 2}\right),
$$

where in $\Sigma_{X}^{\prime} \chi$ runs over all nonprincipal characters mod $q$.

$$
\int_{T}^{T+H}\left(\sum_{\chi}^{\prime} S(t+h, \chi)-\sum_{\chi}^{\prime} S(t, \chi)\right)^{2 k} d t
$$

$$
\begin{aligned}
= & c(k)(\phi(q)-1)^{2 k} H(2 \log (2+h \log T))^{k} \\
& \cdot\left(1+O\left((A k)^{3 k}(\phi(q)-1)^{-1}(\log (2+h \log T))^{-1 / 2}\right)\right)
\end{aligned}
$$

uniformly for $h$ satisfying $0<b \leq\left(H-(H / \sqrt{T})^{1 / 8}\right)$, where $A$ is some positive absolute constant.

(iii) $\int_{T}^{T+H}\left(\frac{S(t, \chi)+S(t+h, \chi)}{2}\right)^{2 k} d t=c_{k} H(\log \log T)^{k}+O_{k}\left(H(\log \log T)^{k-1 / 2}\right)$

if $b \log T \rightarrow 0$ as $T \rightarrow \infty$.

(iv) For any $\Delta$ satisfying $\Delta \log T \rightarrow 0$ as $T \rightarrow \infty$, there exists a function $\Phi(T)$ satisfying $\Phi(T) \rightarrow \infty$ as $T \rightarrow \infty$ such that

$$
\int_{T}^{T+H}\left(\frac{1}{\Delta} \int_{t}^{t+\Delta} S(u, \chi) d u\right)^{2 k} d t=c_{k} H(\log \log T)^{k}+O_{k}\left(H(\log \log T)^{k} / \Phi(T)\right)
$$

Also

$$
\begin{aligned}
\int_{T}^{T+H}\left(\frac{1}{\Delta(\phi(q)-1)} \sum_{x}^{\prime} \int_{t}^{t+\Delta} S(u, \chi) d u\right)^{2 k} d t & \\
& =c_{k} H(\log \log T)^{k}+O_{k}\left(H(\log \log T)^{k} / \Phi(T)\right) .
\end{aligned}
$$

(v) $\int_{T}^{T+H} S(t, \chi)^{2 k} d t=c(k) H(\log \log T)^{k}+O_{k}\left(H(\log \log T)^{k-1 / 2}\right)$.

\section{REFERENCES}

1. A. F. Lavrik, An approximate functional equation for Dirichlet $L$-functions, Trudy Moskov. Mat. Obš̌. 18 (1968), 91-104 = Trans. Moscow Math. Soc. 18 (1968), 101-116. MR 38 \#4424. 
2. A. Selberg, Contributions to the theory of the Riemann zeta-function, Arch. Math. Naturvid. 48 (1946), no. 5, 89-155. MR 8, 567.

3. - Contributions to the theory of Dirichlet's L-functions, Skr. Norske VidAkad. Oslo I 1946, no. 3, 2-62. MR 9, 271.

SCHOOL OF MATHEMATICS, INSTITUTE FOR ADVANCED STUDY, PRINCETON, NEV JERSEY 08540 